\title{
Intervening in the Intergenerational Transmission of Trauma by Targeting Maternal Emotional Dysregulation With EMDR Therapy
}

\author{
Mii Okawara \\ Tokyo Gakugei University, Tokyo, Japan \\ Sandra L. Paulsen \\ Bainbridge Institute for Integrative Psychology, Bainbridge Island, Washington
}

\begin{abstract}
Many studies have shown that the relationship between child and caregivers is critical for healthy development of the child's brain function, and for intergenerational transmission of attachment. Here, we propose a useful eye movement desensitization and reprocessing (EMDR) target for treatment of maternal emotional dysregulation that can cause maltreatment of the child, with the goal of interrupting intergenerational transmission of attachment trauma. First, we use schematics to demonstrate a hypothetical model of the interaction between a child's attachment behavior and a mother's bonding behavior. This schematic shows that the child's physiologic reaction activates or triggers the mother's negative affect and somatic sensations in her limbic system and brain stem and that, in turn, evokes the mother's maltreatment behavior. The negative affect and somatic sensation (maternal emotional dysregulation) are Dysfunctionally Stored Information (DSI) that was produced in the mother's past experience. We propose that the mother's negative affect and somatic sensations activated by the child's behaviors can be useful targets for EMDR therapy based on this hypothetical model. Two Japanese case reports (mothers with 4-year-old daughters) are described to illustrate this application. Further discussion highlights the feature of Japanese cultural relationship and the dissociation, and the meaning and scope of targeting maternal emotional dysregulation with EMDR therapy.
\end{abstract}

Keywords: maternal emotional dysregulation; intergenerational transmission; attachment; Dysfunctionally Stored Information (DSI); EMDR targets

M any studies have shown that child abuse and attachment-related trauma have negative effects upon the child's neurobiological growth, produce considerable emotional dysregulation in children, and lead to mental health problems in adulthood (e.g., Bremner, 2003; Lyons-Ruth, Pechtel, Yoon, Anderson, \& Teicher, 2016; Nemeroff, 2004; Perry \& Pollard, 1998; Schore, 2003; Schuder \& Lyons-Ruth, 2004; Teicher et al., 2003; van der Kolk, 2005). Since Bowlby's seminal tomes on attachment and loss (Bowlby, 1940, 1969, 1973), it has been understood that attachment experience is an interplay between a specific biological/genetic endowment and an environmental context, and that adult psychopathology results from impairments in the original mother-child attachment relationship.
Whereas Bowlby's initial inquiries were into the child's internal world, subsequent research examined the dyadic interplay (Ainsworth, Blehar, Waters, \& Wall, 1978; Main \& Solomon, 1986). Attachment theory proposes that transmission of patterns of relating across generations, including maltreatment, is mediated by an individual's internal working models (Zeanah \& Zeanah, 1989). There are many studies about intergenerational transmission of attachment using Adult Attachment Interview (George, Kaplan, \& Main, 1985) and Strange Situation Procedure (Ainsworth et al., 1978; Main \& Solomon, 1986) (e.g., Berthelot et al., 2015; Cassibba, Coppola, Sette, Curci, \& Costantini, 2017; Rosso \& Airaldi, 2016; van IJzendoorn, 1995). 
A child's neural growth requires that the attachment system be engaged by both nurturing behavior and playful interaction (Panksepp, 1998). At the same time, psycho-biological attunement with the caregiver's interaction system is needed for the child's development of affect regulation (Schore, 2003). In short, to prevent attachment-related traumas from being transmitted generation to generation, it is important to help mothers who cannot build a secure base and functional attachment system or provide good parenting for their children. "As long as the caregiver's memory system remains unprocessed and unintegrated, the child may continue to be exposed to the same experiences that laid the foundation for the development of dissociative mechanisms" (Gomez, 2012, p. 138). In order to improve a mother's capacity for future healthy relationships, her unresolved early maladaptive attachment learning, object relations, and relationship templates need repair. This improved parent-child relationship will then enable her children to develop the capacity for healthy affect regulation and intersubjectivity (Trevarthen, 1998) and to reach other critical milestones (e.g., Malik, 2012; Petzoldt, Wittchen, \& Martini, 2015).

In eye movement desensitization and reprocessing (EMDR) therapy, attachment trauma is recognized as an important aspect of case formulation (Leeds, 2016, p. 75), and this conceptualization is taught by many EMDR therapists (e.g., Adler-Tapia \& Settle, 2017). An EMDR attachment trauma protocol, based on attachment theory, has been offered previously (e.g., Wesselmann, Schweitzer, \& Armstrong, 2016; Zaccagnino \& Verardi, 2016). Many traumatic experiences are addressed in EMDR treatment, by processing the explicit memory of unresolved early experience. However, attachment experience is held in the mysterious realm of implicit memory. O'Shea (2009) and Paulsen (2017) have proposed a protocol of modified EMDR therapy to address trauma held in implicit memory that involves repairing the effects of very early trauma over the first several years of life.

\section{Hypothetical Model of the Interaction System Between Mother and Child}

It is evident that intervening in disrupted mother-child attachment experience is a promising and even critical area for prevention of child abuse, intergenerational transmission, and resulting adult psychopathology. Maternal emotional dysregulation may occur from unprocessed memories (Dysfunctionally Stored Information; DSI) in the mother's past life that are evoked by child-rearing stimuli as explained by the Adaptive
Information Processing (AIP) model (Shapiro, 2001). Negative beliefs, emotions, and sensations related to the numerous and repeated traumatic events experienced by children may be dysfunctionally stored in the brain in memory networks according to attachment theory and the AIP model (Shapiro, 2001; Verardo \& Zaccagnino, 2016). In AIP theory, "the model regards most pathologies as derived from earlier life experiences that set in motion a continued pattern of affect, behavior, cognitions, and consequent identity structures" (Shapiro, 1995, p. 14).

We propose an EMDR targeting strategy for the treatment of disturbed mother-child relationships in order to intervene in intergenerational transmission caused by DSI, and to promote affect regulation and a secure self and healthy relationship. We present the schematic of a hypothetical model of the interaction system between a child's attachment behavior and the biological mother's bonding behavior. This hypothetical model, which includes past attachment theory, is an interaction model focused on maternal emotional dysregulation. Then, we discuss the procedures for targeting maternal emotional dysregulation with EMDR therapy to intervene in the intergenerational transmission of trauma, which are explained. Next, the cultural dimension of Japanese parent-child relationship is explained, and cases of two Japanese mothers are described. We demonstrate that targeting maternal somatic sensations in EMDR therapy is efficacious.

\section{Hypothetical Model}

A biological mother and child are emotionally tied by a biological bond. According to Klaus, Kennell, and Klaus (1995), the term "bonding" refers to the tie from parent to child, whereas the word "attachment" refers to the tie from child to parent, although these have shared characteristics. A functional parent-child relationship is constructed through the interaction between attachment and bonding. Here, we describe the maternal-infant interactive relationship. Figures 1 and 2 are a hypothetical model by which the interactions of the biological mother-child relationship are schematized from the perspective of brain function as described by Tucker, Luu, and Derryberry (2005). Figure 1 shows the functional interaction system model and Figure 2 shows the dysfunctional one.

Functional Interaction System Model. As soon as a negative affect and somatic sensation (as protective reactions) are provoked by the child's limbic system and brain stem, the child calls to help for his/ her mother by vocalization. This is the attachment 


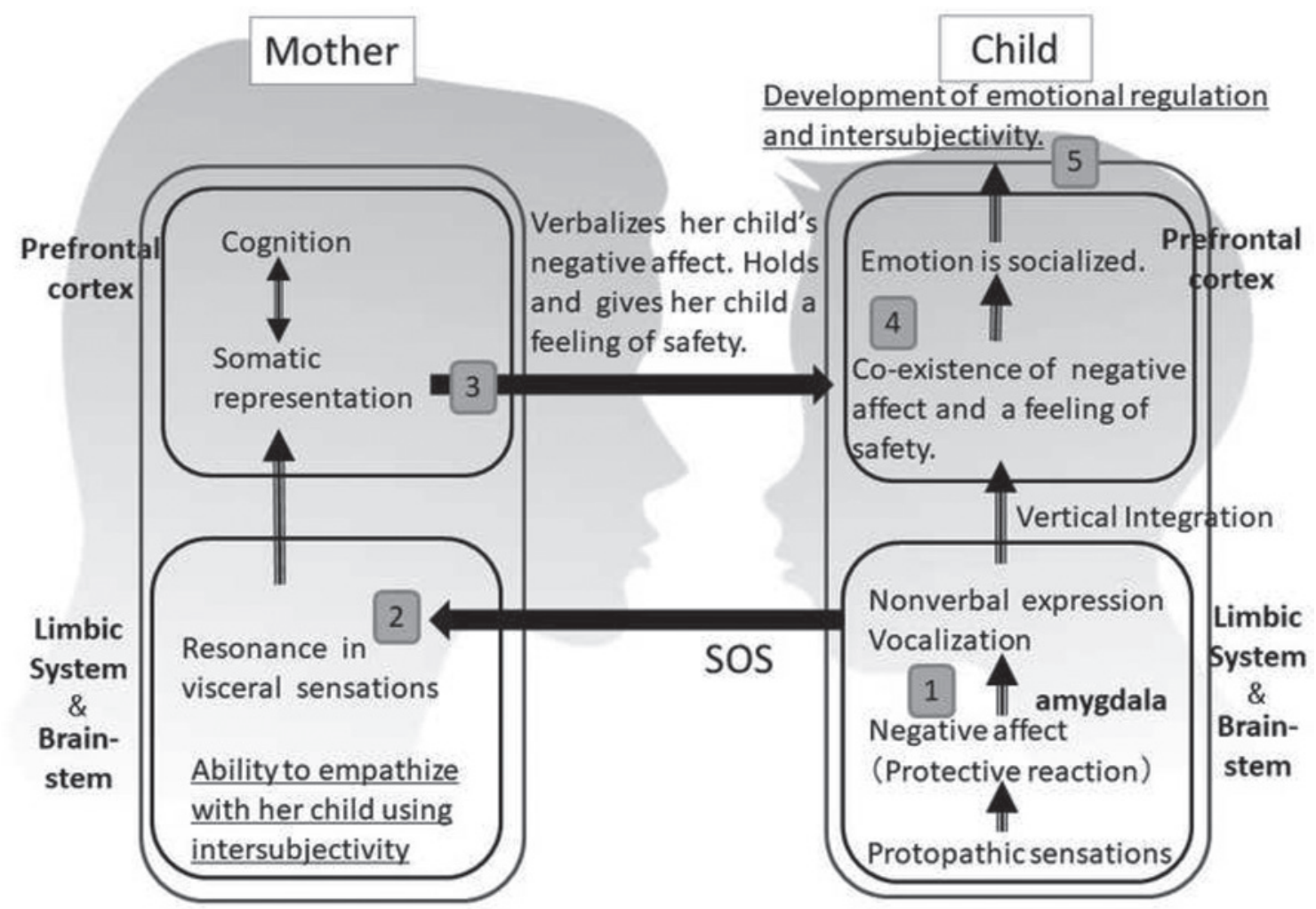

FIGURE 1. Functional interaction system between the biological mother and her child.

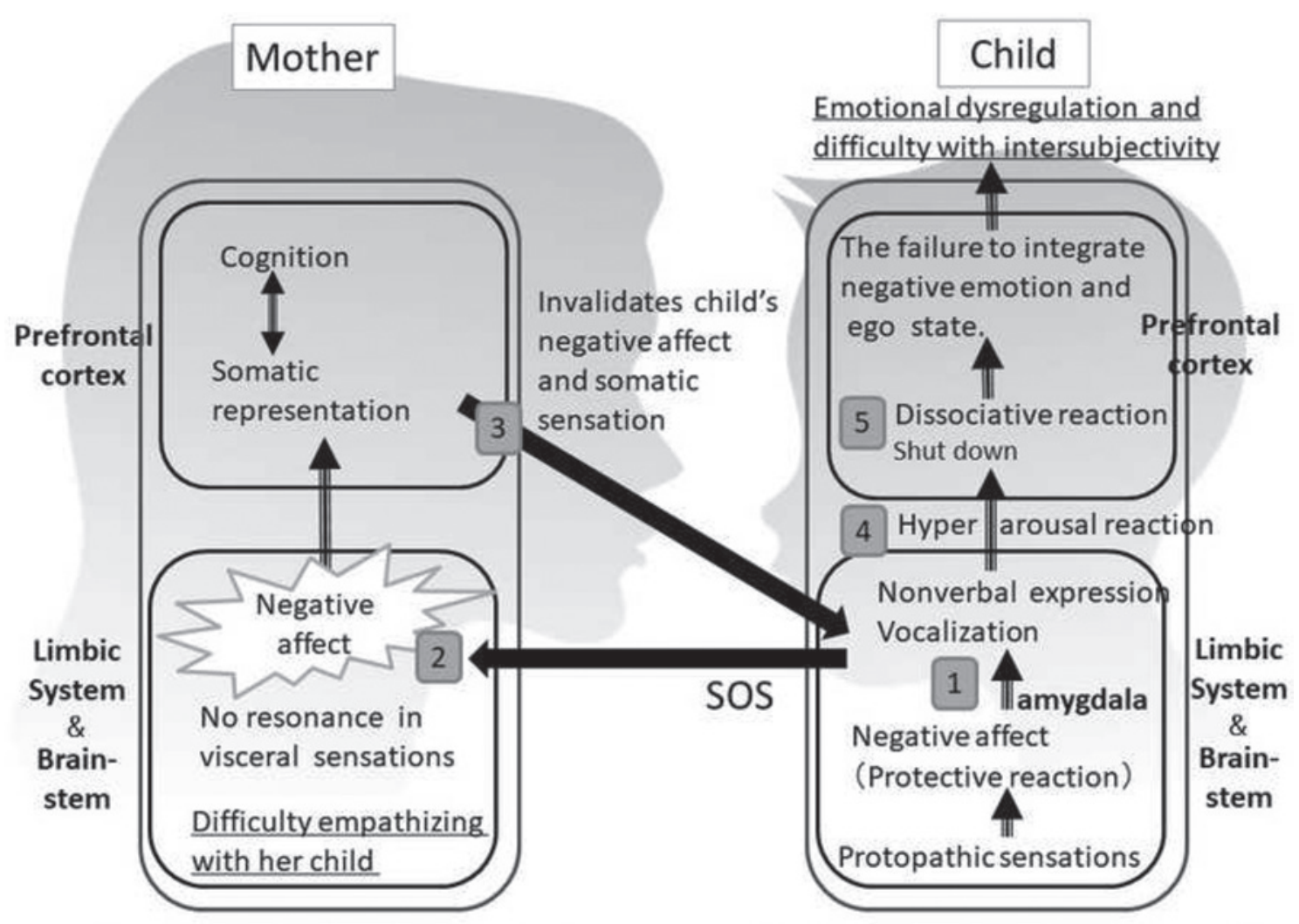

FIGURE 2. Dysfunctional interaction system between the biological mother and her child. 
behavior. Tucker et al. (2005) state that the evolution of empathic concern and social bonding evolved through the encephalization of nociceptive capacity and the evolution of neural mechanisms that integrate pain/distress motive with vocalization lays the foundation for understanding the vertical integration of attachment and regulation of parent/infant bonds (Figure 1, box 1).

The child's crying and fretting as expressed by the evocation of the PANIC circuit lead naturally to the mother's evoked CARE and resonance in terms of visceral sensation in her limbic system and brain stem (Panksepp, 1998). Panksepp (1998) capitalizes these circuits to indicate they are hardwired, present from birth, and require no learning. This reaction is consistent with the affect attunement (Stern, 1985) observed during mother-infant bonding (Figure 1, box 2).

A mother can discern her child's needs through her own evoked resonance as expressed in her felt visceral sensation, so that she can automatically implement the appropriate maternal caretaking behavior as a somatic and behavioral expression that will provide the secure basis her child needs. This discernment of her body sensation and problem solving is the task of the cortex. The mother can make her child feel secure by holding and soothing her or him; she can also empathically verbalize her child's negative affect. Within the language of childhood attachment classification, this relationship is described as the secure group in the Strange Situation Procedure (Figure 1, box 3).

This schematic illustrates the child's state of comfort that is evoked and regulated when the feeling of security arises in the child's brain. The mother's loving gaze evokes a feeling of security in her child, and is thought to activate the child's mirror neurons, thus developing the child's ability to empathize with this mutual intersubjectivity (Trevarthen, 1998). "The simulation of actions in mirror neurons described above may be the somatic analogue to the visceral response of emotional contagion. There is a matching or synchronization of the internal response with the observed actions" (Tucker et al., 2005, p. 709) (Figure 1, box 4).

In the child's cortex, emotional socialization is developed by the process by which the negative affect and the somatic sensation are verbalized. Many research studies have shown that the parent-child relationship affects the development of the child's emotional regulation (e.g., Berlin \& Cassidy, 2003; Edwards, Shipman, \& Brown, 2005). Montag and Panksepp (2017) state that the primary emotional system influences human personality from the viewpoint of an evolutionary perspective (Figure 1, box 5).

Dysfunctional Interaction System Model. Figure 2 shows the dysfunctional interaction system model. This model focuses on the fact that the negative affect and the somatic sensation in the mother's limbic system and brain stem are activated by her child's crying and fretting.

The child's crying and fretting cause unpleasant visceral sensations associated with the negative affect and somatic sensations in the mother's limbic system and brain stem; the appropriate affect attunement to her child's needs, therefore, does not occur. In this article, the cause is understood as maternal emotional dysregulation associated with unprocessed past memories (DSI) (Figure 2, box 2).

The mother acts to regulate the negative affect and the somatic sensations in her own limbic system and brain stem. Some mothers become irritated and scold, neglect, avoid, and reject the child, whereas others are afraid of the child's crying and do everything the child demands. This relationship correlates with three insecure (avoidant, resistant-ambivalent, disorganized-disoriented) groups in the childhood attachment classifications from the Strange Situation Procedure (Figure 2, box 3).

The child experiences a crisis and hyperarousal reaction or another defensive fight-or-flight response (Perry \& Pollard, 1998), with an activation of the PANIC circuit, the basis of child separation distress (Panksepp, 1998). The Strange Situation Procedure has shown that young children in the insecure group are avoidant, resistant-ambivalent, or disorganized-disoriented for their own mothers. Early life stress may shape the development of brain areas, especially the amygdala and hippocampus, involved with emotion processing and regulation (Hanson et al., 2015). Disorganized attachment in infancy predicts greater amygdala volume in adulthood (Lyons-Ruth et al., 2016) (Figure 2, box 4).

Initially, the child's hyperarousal reaction escalates due to activation of the sympathetic nervous system, but once the mother is nonresponsive to the child's distress, the child's reaction is to shut down as a function of the dorsal vagal nervous system as explained by polyvagal theory (Porges, 2007). This response is predicted by Bowlby, who described the infant's sequential responses to separation from the primary attachment figure as protest, despair, and detachment. This sequence includes what is called the freeze response (Perry \& Pollard, 1998). If unrepaired and chronic, the shutdown and collapse become a primary 
structural dissociation so that the child can adapt to the environment (van der Hart, Nijenhuis, \& Solomon, 2010). Negative emotions and somatic sensations that are dissociated cannot be resolved or spontaneously integrated into the child's self (Figure 2, box 5).

\section{The Model, Illustrating the Developmental Pathway to Dissociation}

Dutra, Bureau, Holmes, Lyubchik, and Lyons-Ruth (2009) conducted a prospective longitudinal study of a developmental pathway to dissociation. They stated that dissociation may develop as the result of family relational factors other than physical or sexual abuse. The reasons family communication might contribute to dissociation are illustrated in Figure 2, box 3, which illustrates the effect of invalidation by the mother of the child's biological needs on the child's limbic system and brain stem. Because the child cannot live without the parent's love, the child may be in a double-bind state (Bateson, 1972) that disrupts information processing in the child's brain. The doublebind theory describes the impact of contradictions between information on a context level and information on a content level in communication. Figure 2, box 3 illustrates the effects of contradictions between negative emotions and somatic sensations (information on a content level) and cognition as the meaning of the negative emotions and somatic sensations (information on a context level) in the child's brain. Dissociation can resolve the double bind in the face of the contradiction between the limbic system and the prefrontal cortex to enable the child to adjust to his/her family system and to secure the biological and emotional imperative of being loved by his/her parent.

\section{The Model, Illustrating the Self-Regulation Intergenerational Transmission}

This schematization shows that maternal emotional dysregulation (Figure 2, box 2), that is, mother's negative emotion and somatic sensation, is the motivation of maltreatment for the child. Bridgett et al. (2015) demonstrated a self-regulation intergenerational-transmission model. They state that parent self-regulation exerts strong effects on variations in child-rearing contexts and children's genetic factors, which intersect with these stressful contexts via gene-environment interplay. Intergenerational transmission of abuse, violence, and addiction may be the outcome of poor self-regulation and intergenerational transmission.

\section{Target for EMDR Therapy}

EMDR is a therapeutic approach first developed by Shapiro (2001) to reduce symptoms associated with posttraumatic stress disorder (PTSD). The therapist applies bilateral stimulation (BLS) through lateral eye movement, or alternating ear tones or taps on the hands or knees while the client simultaneously accesses the traumatic memory though image, cognitions, affect, and sensation. A specific eight-step protocol moves the client's therapy through case conceptualization and preparation, desensitization, and reprocessing of the traumatic memory, closure, and follow-up (Shapiro, 2001; Wesselmann et al., 2012).

Leeds (2015) explains that "the target was always viewed as a memory of a specific past incident, current cue, or future occurrence in the first decade of EMDR's development," but many alternative EMDR protocols of which the targets are intrapsychic experience, emotion, urge, and defense have been developed. Some of these include RDI (Resource Development and Installation) (Leeds, 2001); Affect Tolerance Protocols (Leeds, 2013); the Early Trauma approach, which targets attachment experience by time frame, (O'Shea \& Paulsen, 2007; Paulsen, 2014; 2017); DeTUR (Desensitization of Triggers and Urge Reprocessing) (Popky, 2005); Ambivalent (conflicted) avoidance (Knipe, 2010); and the Breastfeeding and Bonding EMDR Protocol (Chiorino et al., 2016).

Originally, Shapiro (2001, p. 50) stated, "It appears that any manifestation of the stored information can be used as a target for the EMDR session." Gonzalez, Mosquera, Leeds, Knipe, and Solomon (2012) explained the importance of focusing on DSI and "the untold." "The untold" is understood as nonrealization. The target proposed here, and the processing procedure, is useful for accessing DSI and "the untold."

As mentioned previously, and according to the AIP model, maternal emotional dysregulation may occur from unprocessed memories (DSI) in the mother's past life that are evoked by child-rearing stimuli. A child's fretting and crying may evoke a hyperaroused sympathetic nervous system response in the mother's limbic system and brain stem, leading to maternal maltreatment of the child (Figure 2, box 2). These evoked states can be understood as affective and somatic flashbacks from the mother's own unresolved trauma history. They could be caused by the mother's childhood memories, ego states, or the chain of negative emotions and somatic sensations linked to those memories. 
Our model proposes targeting the biological mother's negative emotions and somatic sensations (maternal emotional dysregulation) activated by her child's crying and fretting (Figure 2, box 2) during EMDR therapy.

\section{Japanese Cultural Feature About Parent- Child Relationship and Dissociation}

In this article, this hypothetical model is examined with Japanese cases, so cultural features of Japanese parent-child relationship and dissociation are explained in the text that follows.

In any culture, the parent-child relationship unconsciously defines the individual's ego boundaries. The relative importance of individual vs collective identity vary depending on the specific culture and the history by which the society is constructed. American anthropologist Benedict (1946), described in her famous book The Chrysanthemum and the Sword: Patterns of Japanese Culture that Japanese culture is "shame culture" in regard to "guilt culture" in the Western world.

For example, an ego boundary of Western individual identity is like a peanut with the husk and a Japanese ego boundary is like a peanut without the husk. Japanese "husk" is the boundary of his/her shared group: family, school, company, community, and so on. The relationships of the shared group, which construct the hierarchy, define the self of Japanese. Therefore, the concept of $w a$ in Japanese is related to the seminal role of shame in Japanese culture in general. With $w a$ it is understood that a person should always consider what others in the group need to feel comfortable. This shared understanding is important in Japanese society, so in Japan the expression of negative emotions in the group is recognized as poor behavior, though in Western culture it might be considered virtuous because it is assertive. Indeed, it is a primary Japanese need to make paramount and respect the needs and feelings of others in the group. For an individual to have needs and feelings for that individual's sake, without consideration of the others' needs and feelings, is considered selfish. Selfishness is understood to be shameful, so an individual who selfishly makes his or her own needs and feelings paramount likely feels shame in relation to the group.

Beyond infancy, as children get slightly older, Japanese mother-child ego boundaries under these cultural situations are blurred, compared to Western ego boundaries, which are more individualistic. What might be considered enmeshment or lack of individuation in Western culture is a commonly held value in Japanese culture well into child development. Doi (1971), a Japanese psychoanalyst, published the cultural theory about this Japanese feature in the United States. He presented the concept of amae, the Japanese word that means attachment behavior, as a fundamental construct foundational to Japanese culture and society. Of course, attachment phenomena are observable in human babies in all cultures, and Bowlby developed the attachment theory to be universal. However, there are no English words for the Japanese concept of amae because amae is the culturally Japanese phenomenon of shared ego boundaries between mother and child. The Japanese mother can readily notice her child's needs without her child's demand through mutual wordless anticipation because their boundary is blurred, as if they have a shared self. One benefit of this cultural phenomenon is the high empathizing abilities in Japanese mothers.

However, the cultural differences in ego boundaries might produce a difference in dissociative barriers as well, especially as it relates to inhibition or expression of negative affect. According to the first author's clinical practice in Japan (Okawara, 2012, 2015), a Japanese dissociative child and his/her parental interactions have two mutually exclusive outcomes or phases. One phase is the absence of expression of the child's negative affect, and the other phase is the expression of the child's negative affect without consideration of the parent's needs and feelings. In response to the former, the mother utilizes her high empathizing ability and demonstrates deep love for her child by anticipating the child's needs and feelings. In response to the latter phase, the mother invalidates the child's negative affect and somatic sensations due to her own unprocessed attachment/trauma history and also the cultural reasons mentioned previously. Although this would normally be understood to be disorganized attachment because it is inconsistent, in Japanese culture, it is more organized, though phasic in nature. Because these two alternative phases are not mutually consistent or compatible, the result is primary dissociation in order to hold these two disparate phases sequestered, and it enables the child and parent to accommodate societal expectations. We assert that the irreconcilable double bind is enough to create the structural dissociation even though there is no physical and sexual abuse.

As previously mentioned, the Japanese self is constructed in relation to other persons. Therefore, Japanese persons who developed their self with dissociated negative emotions and somatic sensations in order to adapt to their parental expectations are recognized as "good children" and "good persons" in 
their school and society, and they are highly praised over time. As they have the aspect of self that was raised in high empathizing care, they can live without pathological symptoms even though they have plural dissociative negative emotional parts or ego states. However, after the Japanese woman who was "a good girl" in childhood becomes a mother, her DSI memories and plural negative emotional parts evoked by her child's voice can lead to child-rearing difficulties and mistreatment of her child. Although these mothers are not otherwise mentally ill, their capacity to engage lovingly with their children is impaired on a daily basis. The parent invalidates her child's negative affect and somatic sensations because they have memories, whether explicit or implicit, of their own parents invalidating their negative affect and somatic sensations. This is the intergenerational transmission of attachment trauma.

The cases in this article involve Japanese biological mothers, whose complaints are maternal emotional dysregulation. They report no other pathological symptoms and function well within Japanese culture, generally.

\section{Case Illustrations}

Two Japanese case examples are provided to show that effective recovery of the mother-child dysfunctional interaction system can be achieved by targeting the biological mother's negative emotions and somatic sensations (maternal emotional dysregulation) activated by her child's crying and fretting (Figure 2, box 2) during EMDR therapy. All names have been changed. Throughout, the therapist referenced in the cases is the first author. The standard eight-phase model of EMDR therapy and the three-pronged protocol are used, except in the way in which the target is identified.

The process of identifying the target is as follows. The EMDR therapist listens empathically to the mother's narrative about her own emotional dysregulation to achieve a sense of safety in the therapeutic relationship. The therapist attunes empathically with the mother's negative emotions and somatic sensations that are evoked by the child's behavior, as told through the mother's narrative. The therapist asks her, "How different would you feel, if you did not have the negative feeling and body sensations in the presence of the child's behavior?" The therapist explains that it is possible to resolve those uncomfortable body sensations and emotions by using EMDR therapy to work with the mother's unresolved memories.
In the first EMDR session (conducted within the secure treatment relationship), the therapist guides the mother to notice any somatic sensations with breathing, while remembering the child's behavior as stimuli. The therapist explains that this is the mother's own brain response. A Subjective Unit of Disturbance (SUD) score is determined by asking the mother to rate her level of distress on a $0-10$ scale. Subsequently, the therapist helps the mother achieve a safe state through self-tapping and breathing while remembering a positive memory with her child or through the use of another positive resource. The therapist teaches the mother to use this stabilization method in her home. The preparation stage of the following cases is omitted in this description.

\section{Case Report 1 (Mother's name is Aiko, 35 Years Old)}

Aiko's 4-year-old daughter Miki was a very bright little girl. Her family consisted of four people: Aiko, a husband, Miki (4 years old), and a son (2 years old). Aiko visited the therapist's office because Miki had started to pull out her own hair, evidencing trichotillomania, and was reluctant to go to kindergarten. Aiko had four psychotherapy sessions over a period of 4 months. Aiko and her daughter, Miki, each had one EMDR session. The EMDR successfully improved Miki’s trichotillomania.

History Prior to Treatment. When Miki was 2.5 years old, her younger brother was born. Thereafter, Miki had developed atopic dermatitis and begun furiously to scratch her skin every night when her brother was being breastfed. When she was around 3 years old, she became increasingly jealous of her brother. When Aiko found Miki hitting her brother while straddling him, she pulled Miki's arm to move her away, unintentionally dislocating Miki's arm. Because taking care of two children at home was quite a burden for Aiko, Aiko and her husband enrolled Miki in kindergarten when she was 3 years and 3 months old. When she was 4 years old, at the end of the summer vacation, she refused to go to kindergarten and her atopic dermatitis worsened. After a triggering event in which Aiko scolded Miki for scratching her skin, Miki could not stop pulling out her hair. While at kindergarten, she did not cry or become upset but was overly well behaved. In contrast, at home, she scratched her atopic skin furiously because of stress. Aiko became irritated at Miki and, consequently, Miki's atopic dermatitis and trichotillomania progressed. They became trapped in a vicious circle. In contrast, Aiko's husband treated their 
children gently, understood Aiko's difficulties related to taking care of the two children, and spent holidays with their children.

EMDR Therapy for the 4-Year-Old Daughter, Miki. At the first session, Miki showed variable ego states: one moment she appeared like a 1-year-old child when held on her mother's lap, and the next moment she spoke like a bright 4-year-old girl. As Aiko spoke of the episode in which Miki's elbow was inadvertently dislocated, the daughter appeared to dissociate, as evidenced by rapid respiration and dull eyes. The therapist then told Aiko to hold Miki tightly and provided EMDR therapy. The mother's close holding of her daughter provided a safe place. After the EMDR, Miki's days became more peaceful and she pulled out her hair less frequently, except when Aiko scolded her.

EMDR Therapy for the Mother, Aiko. At the first session, Aiko talked about her family of origin: She had a brother 3 years older and had grown up in an ordinary but old-fashioned Japanese family in which the eldest son was treated as the most important.

Aiko was suffering from the fact that when she saw Miki furiously scratching her atopic skin she became irritated and felt a hot surge of anger. Therefore, as targets for EMDR, I used the emotion and somatic sensation that occurred when Aiko saw Miki furiously scratching. Aiko's negative cognition (NC) was "I'm incompetent"; her emotion was "anger"; and her somatic sensation was "I feel pressure in my head" $(\mathrm{SUD}=8)$. When knee taps were added as BLS, Aiko said that she had seen a child with atopic dermatitis being bullied and that as a mother she was concerned about her inability to do anything for her daughter's atopic dermatitis. She then burst into a flood of tears. After the desensitization process had been completed, in a relatively short time, Aiko said, "Now, I'm feeling good." The positive cognition (PC) at the end of the session was, "I'm just who I am."

One month later, Aiko reported that she had stopped getting irritated by the behavior of her daughter scratching her skin and had become able to properly deal with fussy children in her everyday life. Aiko looked back on what had happened to herself. When she saw Miki furiously scratching her skin, she felt as if she had heard a voice saying, "You're incompetent." After she realized that the words were those of her mother, who had repeatedly said them to her since her childhood, she stopped getting irritated. Because the relationship with her mother was pretty good at the time of the session, the childhood conflict with her mother became more neutral.
Following are the memories that Aiko recalled after the EMDR: After years abroad from ages 1 to 5 years, Aiko returned to Japan. When she was talking with her brother in English, she was bullied by her peers. This experience taught her that she should not be herself. Aiko's mother was a perfectionist; she denied everything Aiko did and made her do it again. When Aiko was angry about her classmates at elementary school, her mother told her, "Your display of anger is inappropriate. If you always get angry, you will abuse your children." Aiko thought of herself as an evil person and felt frightened of herself. Her brother, who was 3 years older, had secluded himself from society and stayed at home for 8 years after entering college. Although he had worked constantly since graduating from college, he had grown up with significant challenges until he had become independent and developed a solid sense of identity.

Aiko understood her mother's circumstances well. Aiko's mother had lost her own mother when she was 9 years old. After that, she had been bullied by her stepmother and physically punished by her military father. These experiences made her a person with low self-esteem, and she therefore invalidated her children when she was rearing them. Fortunately, Aiko had a good relationship with her mother at the time of the therapy and was able to work out her life issues, including her mother's difficulties.

Seeing her daughter furiously scratching her skin triggered Aiko's childhood memory of being bullied, owing to anticipatory anxiety that her daughter might be bullied because of her atopic dermatitis. This triggered the memory of her mother's words, "Don't feel angry. If you get angry, you'll become an abusive mother," resulting in Aiko's feelings of anger and confusion. A single EMDR session successfully reprocessed these memories so that her past memories were no longer triggered by her daughter's present behaviors.

Two months later, Miki stopped hair pulling, showed stable ego states, and appeared appropriate for her age. Her atopic dermatitis had also improved. Aiko thought that Miki's kindergarten was unsuitable for her and changed her environment to suit their needs, selecting a more easygoing life.

\section{Case Report 2 (Mother's name is Mari, 34 Years Old)}

Mari had lost control of herself after the birth of her daughter. When her daughter cried in a loud voice, Mari became caught up in the fury and scolded her daughter endlessly. Mari's family consisted of three 
people: Mari, a husband, and a 4-year-old daughter. Mari was struggling because she loved her daughter but could not treat her daughter gently as other mothers would. In this case, the period of psychotherapy was 8 months, the number of sessions was six, and EMDR was provided three times to Mari. The emotions and somatic sensations evoked by the daughter's loud voice and selfish behaviors were used as targets in the first two sessions. In the third session, an unresolved traumatic memory that had not been recognized was used as a target. This process successfully solved Mari's problem.

History Prior to Treatment. Because Mari used to be a childcare worker, she had a sound knowledge of child-rearing. However, she could not stop herself from feeling irritated and acting angrily when her daughter cried loudly. Her toes were always clenched. Because her husband was able to treat his daughter gently, the daughter behaved calmly when she was with him. Therefore, Mari thought that there was no problem with her daughter but that the problem was her own background; this prompted her to visit the therapist's office.

Mari's family of origin consisted of four people: Mari, a brother 2 years older, a mother, and a father. Because her brother had been frequently admitted to the hospital for asthma treatment, her mother had been overly protective and had dominated her brother. In contrast, she showed no interest in Mari. Mari still remembered vividly that her mother had repeatedly told her, "You always cried loudly," and that even when Mari had been hit by an indoor shoe at kindergarten and her forehead had been injured, requiring stitches, her mother had told her, "It's your fault." Mari had no memory of playing with her mother, and her father often went out for drinks and was absent from home. When he came back, he was often severely intoxicated. Although she did not actually like him, she behaved as if she did. She thought that that was a good thing to do because people around her smiled and said, "You're a daddy's girl." As long as she tried to be funny, people around her felt happy. When Mari was a fourth grade student at elementary school, her brother was physically punished by being hit with a bamboo sword at junior high school. Out of spite, he behaved violently toward her at home. She was always very scared of him coming home. Although her mother knew about it, she overlooked it because she felt pity for her son because he was struggling academically. After a while, his mood was so depressed that he refused to attend school. Their mother became proactively involved in social activities relevant to school refusal. Although
Mari's brother had refused to attend high school, thereafter, he worked locally and was a successful member of society. When he became an adult, he apologized to Mari for his earlier behavior, so she felt she shouldn't complain to her mother about the unfair treatment. Under these circumstances, Mari thought that she should not bother her mother. She was expected to be independent and able to do everything by herself. By doing so, she was recognized as a dependable daughter, a characteristic that was highly valued as a traditional Japanese virtue.

The First EMDR Session for the Mother, Mari. Mari said that when she heard an annoying, loud voice, she could not control herself. The following is a recent episode in which she was confused: With her daughter, she went to an amusement park where popcorn was being sold in the kind of containers that children find irresistible. Because they had already bought something to eat, she told her daughter, "We won't buy popcorn." Then her daughter insisted in a loud voice, "I want the container! I want the container!" The moment Mari heard her daughter's loud voice, she went out of control and kept yelling at her daughter. From this perspective, the therapist selected the emotions and somatic sensations evoked by an annoying loud voice as targets to start EMDR.

Mari's NC was "I can't stand it." Her PC was "I can control my emotions," and the Validity of Cognition (VOC) score was 2 (1=completely false, $7=$ totally true). She reported a sensation of pressure in her head and her emotion was "anger" (SUD = 2). As BLS was added through knee taps, Mari's irritation turned to sadness and she shed tears, saying, "I don't know why I'm crying." The therapist asked her, "How old do you feel, when you feel that somatic sensation?" and she replied, "About 6 years old." The therapist told her to notice the feeling of the 6-year-old girl and applied knee taps. Mari said, "The girl is saying that she wants someone to help her and protect her," indicating that Mari was talking with the 6-year-old ego state. The therapist asked her, "What would your 34-year-old self like to tell your 6-year-old self?" She replied, with tears in her eyes, "You don't need to go out of your way in order to be liked by the people around you." The therapist told her to hold the 6-year-old self and applied knee taps. After a while, when she felt calm, she said, "My previous image was of a 6-year-old self with long hair, but now a fourth-grade elementary school student self with buzz-cut hair is here." The therapist told her to stay there and added knee taps again. Then Mari began to cry, saying, "A little girl is weeping, saying that she's in pain." She recalled the 
memory that she had been physically punished by her classroom teacher for 3 years, from the fourth to sixth grades of elementary school. At that time, because she was a rebellious and difficult student, the teacher often grabbed her by the chest and banged her against the wall. Furthermore, after coming home, she was hit by her brother. Desensitization and reprocessing of this memory proceeded while she was crying. Then Mari realized that her memory of anger, for which she had been physically punished, was evoked when her daughter behaved rebelliously. Mari's PC at the end of the session was, "I can be my true self." She also became aware that the loud voice of her daughter could be her own loud voice, which her mother had disliked, as well as the loud voice of her drunken father.

The episode of physical punishment by her teacher for 3 years from the fourth to sixth grades of elementary school was an untold memory and was unconscious at the first session. Although Mari showed multiple ego states, her present 34-year-old ego state was capable of holding the past younger ego states.

The Second EMDR Session for the Mother, Mari. After the first session, Mari no longer became reactive in the presence of her daughter's loud voice. The somatic reaction (clenched toes), which she had felt for years, also disappeared. However, when her daughter kept a shovel all to herself in the sandbox and insisted that she would not share it with others, Mari could not control her anger and lost her temper. This situation was used as a target of the second EMDR. Mari's NC was "I must be perfect"; her PC was "I'm just who I am" (VOC = 1). Her emotion was "anger," and her somatic sensation was "within the stomach" (SUD = 2). Soon after, as the therapist added knee taps, she complained that her stomach felt tight. The therapist told her to stay with the somatic sensation. She then said, "Please praise me because I keep working so hard. Please praise me because I won't bother you. Please praise me because I'll be dependable." Then she burst into tears. She recalled that she wanted her mother to look at her and therefore worked very hard to be recognized and praised by her. During knee taps, Mari reported that the present-day Mari was holding and speaking to her little self, saying, "Just be yourself." Hearing Mari's words, "Your very existence is a blessing," the little self smiled through her tears. As processing continued, Mari remembered that as an elementary school student, she wanted to die and tried to choke herself to death. Remembering this in tears, Mari asked, "Mom, why do you devote all your attention to my brother? You don't speak to me at all. You always told me I was a dependable girl, so I thought I must be." She said that her younger selves, of various heights, rushed into the arms of her present self, while saying, "Help me! Help me!" She said that her present self took them in her arms and said, "Whenever my daughter has a hard time, I will help and protect her." As they integrated, these younger ego states conveyed to adult Mari that the past was over and complete. Mari's PC at the end of the session was "I deserve to be happy."

Mari realized that she had felt surges of anger toward her daughter because she thought that she would not be regarded as a dependable mother unless her daughter was a warm-hearted girl. Eventually, she accepted that her daughter's behavior was appropriate for her age.

The Third EMDR Session for the Mother, Mari. One month later, Mari reported that she was getting angry less frequently. Although she was experiencing less anger, she had noticed that when she was short of sleep she could not control herself well. The therapist asked her, "When did you have the worst sleep deprivation in your life?" It had been between the ages of 21 and 25 years when she had worked in childcare. The following was her typical daily schedule at the time: After closing the nursery school at 19:30, she worked overtime until 22:00 every day and returned home at 23:00. Then, she drank alcohol to intoxication because of the stress and stayed up late. Next morning, she woke up at 6:00 and went to work. The nursery school, which was very strict and operated under a stern disciplinary policy, tried to control the children with loud voices and to force them to be good children. Following the policy of the nursery school was stressful for her. She was frequently scolded by her supervisor and verbally abused by the principal, who said, "You are a dumb hick!" Every time she remembered this experience, she became filled with fear. On Saturday, she stayed drunk until the next morning, and on Sundays she slept all day long. She showed evidence of a shopping addiction and ran up a considerable debt. During this talk, her fear reached SUD $=10$. The experience at the nursery school was determined to be an unprocessed trauma.

This memory was used as an EMDR target using the standard protocol. The NC was, "I can't stand who I am," and her PC was "I accept myself as I am" (VOC =1). Her somatic sensation was "chest tightness" and her emotion was "fear" (SUD $=10)$. The self who was being scolded for performing poorly at the piano and at the staff meeting was evoked. Her guilt and sadness for imposing the nursery school's overbearing 
instructions on the children and remaining at the job to avoid dependence on her parents were desensitized and reprocessed. The PC that fit best at the end of the session was "I've learned from it." She said, "I realize that it is important to acknowledge my negative emotions even when I must work hard."

She realized that the reason she became enraged when her daughter spoke in a loud voice was because she had not, herself, been permitted to be expressive. She was similarly stifling her daughter's expression and scolding her to protect her from punishment at school.

After processing the trauma, Mari stopped feeling angry toward her daughter and losing control over herself. In addition, other parents at the school said her daughter's facial expressions had become more cheerful and less strained, and her behavior had become calmer.

\section{Results}

\section{Summary of Treatment Process: Case 1}

In Case 1, due to the daughter's atopic dermatitis, which was aggravated by the stress, the daughter had been clawing at her skin and developed trichotillomania. The daughter's behavior had activated the mother's childhood memory, a feeling of confusion, and the cognition "I am a bad mother." The first EMDR session removed this link through desensitization and reprocessing.

Aiko's childhood memory (DSI) in which her mother told her, "You will abuse your child in future because you get angry so easy" came out through reprocessing of negative emotions and somatic sensations activated by the child's scratching behavior. Previously, she had no access to this childhood memory because there was no continuity, linkage, or co-consciousness between her present ego state, which had a good relationship with her mother, and her childhood ego state. One session of EMDR therapy using the target of the somatic sensation and negative emotion was sufficient to process Aiko's memory of her past life, with its DSI, to adaptive resolution, allowing her to restore her original perceptions of her child-rearing ability.

\section{Summary of Treatment Process: Case 2}

In Case 2, during the history-taking session, the mother, Mari, told of a serious and complex problem in her family history, although her daughter had no symptoms. After starting EMDR therapy in which the target was the emotional irritation and uncomfortable somatic sensation activated by the child's behavior, the mother became aware of younger ego states. It is understood that these ego states represent neural networks holding unresolved traumatic experience. Paulsen \& Lanius, 2009, p. 344) further stated:

Ego states may be organized to enhance adaptability in coping with specific events or problems and they can also be delineated by time dimensions, signifying the age at which formation occurred (child, adolescent or adult), affect, cognition, or bodily states, and so on.

The way in which the therapist implemented the desensitization and reprocessing process in Case 2 was similar to the exercise to increase compassion for a hurt child ego state described by Paulsen and Lanius (2009, p. 364):

You can then use this exercise either as an interweave during trauma processing or specifically setting up the EMDR Standard Protocol with the adult holding the child.

The therapist added BLS while keeping a dual focus, promoting the mother's current ego state as an adult mother holding her past child ego state warmly and with compassionate acceptance. In the mother's first and second sessions, she was able to accept her child ego state without resistance. Because of this acceptance of the child state, the DSI from the AIP model was able to be processed to an adaptive resolution.

After two EMDR sessions that enabled her to recover from being irritated by her child, Mari became aware that a lack of sleep caused her to reach a state in which she could not control her emotions. She identified the untold, unprocessed trauma in her ex-workplace by focusing on the memory about the lack of sleep. We understood that the memory about her brother that she had talked about in the session had already processed naturally, so the memory had continuity and sufficient conscious linkages with her present ego state. The untold and unprocessed DSI memories led to several emotionally burdened child ego states and the additional traumatic experiences in her ex-workplace had remained untold and unprocessed.

In this manner, targeting maternal emotional dysregulation enabled natural reprocessing of the unprocessed traumatic memory. Both mothers and their children have maintained recovery from their problems for more than 2 years after the therapy was terminated. 


\section{Discussion}

The Feature of Japanese Cultural Relationship in the Family and the Dissociation

In both cases, the mother was the good daughter/ good sister who helped to maintain the family system in which her brother was the IP (Identified Patient). She was valued highly by her parents as the good daughter who was no trouble in comparison with her brother. The mothers had no apparent symptoms in childhood or at that time when they came to the therapist. However, they had dissociative memories and parts (ego states) that have been unprocessed.

The theory of structural dissociation of the personality (van der Hart et al., 2010) describes two prototypical personality subsystems: EP (the emotional part of the personality) and ANP (the apparently normal part of the personality). The EP is basically fixated at the time of acquisition of unprocessed traumatic memories that frequently involved childhood emotional, physical, and sexual abuse and caretaking problems. The ANP is mediated by action systems for functioning in daily life and commonly seeks the approval of caretakers to gain acceptance, protection, and love (van der Hart et al., 2010).

As previously mentioned, Japanese ego boundaries are different from those of Western individual identity and Japanese ego is constructed in interactions with the member of the shared group (family, school, community, etc.). Therefore, a Japanese ANP could be the normal and socially approved self. According to the first author's experience conducting EMDR therapy for the well-socialized Japanese client without mental illness, during processing, self-acceptance of the mother's own child ego states or plural EPs occurs naturally without resistance. As shown in Case 2, however, sometimes cognitive interweaves are needed for that self-acceptance of the child ego states. We hypothesize that this ready self-acceptance may be due to thin ego boundaries found in external relationships that are replicated in the internal self-structure, with permeable boundaries between self-states. We further hypothesize that many Japanese children easily dissociate emotional parts of self because those emotions and needs, especially if they include negative affect, are invalidated by parents because of social requirements and cultural expectations. It follows that during EMDR processing, EPs could be easily integrated with an ANP (the socialized self), especially if that socialized child is loved by a Japanese mother with the previously described high ability to empathize because of a culturally traditionally thin ego boundary between mother and child. The mechanism of dissociation may be differentially utilized within different cultures based upon varying social contexts about ego boundaries, unspoken expectations, and the evaluation of negative emotion.

\section{The Meaning of Targeting Maternal Emotional Dysregulation With EMDR Therapy}

In this population, mothers who are irritated by their children and engage in abusive behavior with them do not present with the chief complaint of their own unresolved explicit trauma history, but rather, present with complaints about their child's symptoms, suffering, or the mother's vexation with the child. They are in trouble because they feel they cannot be "good mothers" due to maternal emotional dysregulation. This situation thus reminds them of their own life stories and evokes or exacerbates their low self-esteem and dysphoria.

Neither mother presented for individual therapy, but rather presented only with concerns for their child and child-rearing, although they had, themselves, dissociated experiences that contributed to their child-rearing issues. Those unresolved dissociated experiences were only evoked by the demands of child-rearing, which triggered a related effect on the mother's capacity for emotional self-regulation. This is the seminal finding of this case study, that it is only in the mother-child interactive relationship that the mother's unprocessed experience becomes triggered and can result in both attachment injury and child abuse. The primary structural dissociation is always a function of adjustment and accommodation to the requirements of the environment. However, the locus of the problems of child-rearing and child abuse are in the DSI, which holds the negative emotions and somatic sensations evoked only through the experiences of birth and child-rearing. These negative emotions and somatic sensations can be targeting during EMDR therapy to effectively intervene in a conflictual attachment relationship between mother and child. It is better to target the focal somatic sensation of irritation or other disturbance than to presume a specific explicit memory. This is because the mother's unresolved early attachment disturbance or the untold and unprocessed DSI memory may be held in implicit memory. The choice of a somatic experience as an EMDR target may lead directly to accessing and processing maternal attachment trauma and previously unprocessed traumatic memory held in implicit memory, even in the absence of explicit memory or narrative. 
Only the brain can know, and not consciously, which memory is linked with which emotion or somatic sensation. The therapist cannot predict how memories are processed in relation to the target, namely, the negative emotions and somatic sensations activated in the mother by the child's behavior. EMDR processing automatically catalyzes the client's neural networks holding the unvoiced and unprocessed DSI memory through targeting of the negative emotions and somatic sensations underlying the abusive behavior. The chain of associative linkages engaged in by the brain during EMDR processing outperforms a therapist's modest attempts at surmisal of the causal origins of the disturbance.

\section{Scope of Targeting Maternal Emotional Dysregulation With EMDR Therapy}

These two cases illustrate how EMDR therapy can target and process the negative emotions and somatic sensations in the mother that are activated by the child's behavior. The two mothers loved their children very much, but they were having trouble with child-rearing because of their negative emotions and somatic sensations. They felt that their marriages were very happy and their relationships with their husbands were good, and both husbands were calm fathers to their children. The mothers also had good relationships with their families of origin at the time of the therapy.

If a client with problems in child-rearing has problems in her marriage and her relationship with her husband, or if her husband cannot function as a father, then integrative therapy (EMDR therapy and family therapy) should be planned from a long-term perspective (Okawara, 2010, 2012). In such situations, using this target in EMDR therapy sometimes makes the mother aware that the essential problem is not only child behavior but also a marriage issue because the choice of a somatic experience may lead directly to unprocessed DSI held in implicit memory.

If a client with problems in child-rearing presents for individual therapy with pathological symptoms, she should naturally be treated with a treatment plan that targets those symptoms. Zaccagnino and Cussino (2013) present a clinical case report of a mother who was referred to treatment because she presented strong depressive symptoms exacerbated after the birth of her child. In this case, the mother's depression was so strong that her partner was afraid that she might kill the baby. When the mother has pathological symptoms and is not stable, the maternal trauma issue should be treated along with therapy to enhance the client's parenting skills.

If, in contrast, the mother is traumatized in pregnancy, childbearing, or the perinatal period, she may have difficulties loving her baby because of maternalchild bonding failures (Klaus et al., 1995). In such cases, EMDR therapy could target traumatic memories of the pregnancy, childbearing, and the perinatal period (De Divitiis \& Luber, 2016; Madrid, 2007; Madrid, Skolek, \& Shapiro, 2006).

If the biological mother has distress related to breastfeeding, the therapist can use the Breastfeeding and Bonding EMDR Protocol (Chiorino et al., 2016). The Breastfeeding and Bonding EMDR Protocol focuses on the moments of greatest difficulty/distress related to breastfeeding to rehabilitate the bonding between the biological mother and her baby. EMDR therapy targeting the moments of greatest difficulty/distress in the relationship with her is consistent with our method. The moments of greatest difficulty/distress related to breastfeeding are shown as box 2 in Figure 2.

\section{Limitation of This Article and Future Possibilities}

This proposed model of the interaction system between mother and child is based upon a clinical hypothesis as a result of observing the results of clinical intervention. We suggest further controlled studies to verify the accuracy of our hypothesis that unprocessed attachment traumas related to child-rearing may be wired to the biological mother's negative emotion and somatic sensations as DSI (Figure 2, box 2).

As this study is within a Japanese cultural context, it remains to be seen whether this target is useful for biological mothers in other cultural contexts as well, as there might be cultural and family differences in the identity structures behind the emotions and behaviors. If the finding is generalizable, it would likely be at the point of the biological mother-child relationship.

In this article, we proposed a practical EMDR target for mothers who have a focal complaint about maternal emotional dysregulation. Placing a priority on maternal emotional dysregulation is key to preventing child abuse and attachment-related trauma syndromes. EMDR therapy appears to have a great potential in this global priority.

\section{References}

Adler-Tapia, R. L., \& Settle, C. S. (2017). EMDR and the art of psychotherapy with children: Infants to adolescents. New York, NY: Springer Publishing. 
Ainsworth, M. S., Blehar, M., Waters, E., \& Wall, S. (1978). Patterns of attachment: Assessed in the strange situation and at home. Hillsdale: NJ: Erlbaum.

Bateson, G. (1972). Steps to an ecology of mind. New York, NY: Ballantine Books.

Benedict, R. (1946). The chrysanthemum and the sword: Patterns of Japanese culture. Boston, MA: Houghton Mifflin Harcourt.

Berlin, L. J., \& Cassidy, J. (2003). Mothers' self-reported control of their preschool children's emotional expressiveness: A longitudinal study of associations with infant-mother attachment and children's emotion regulation. Social Development, 12(4), 477-495. http: / dx.doi. org/10.1111/1467-9507.00244

Berthelot, N., Ensink, K., Bernazzani, O., Normandin, L., Luyten, P., \& Fonagy, P. (2015). Intergenerational transmission of attachment in abused and neglected mothers: The role of trauma-specific reflective functioning. Infant Mental Health Journal, 36(2), 200-212. http: / / dx.doi.org/ 10.1002 / imhj.21499

Bowlby, J. (1940). The influence of early environment in the development of neurosis and neurotic character. The International Journal of Psycho-Analysis, 1, 154-178.

Bowlby, J. (1969). Attachment and loss. Vol. 1: Attachment. New York, NY: Basic Books.

Bowlby, J. (1973). Attachment and loss. Vol. 2: Separation. New York, NY: Basic Books.

Bremner, J. D. (2003). Long-term effects of childhood abuse on brain and neurobiology. Child and Adolescent Psychiatric Clinics of North America, 12(2), 271-292. http:/ / dx. doi.org/10.1016/S1056-4993(02)00098-6

Bridgett, D. J., Burt, N. M., Edwards, E. S., \& Deater-Deckard, K. (2015). Intergenerational transmission of self-regulation: A multidisciplinary review and integrative conceptual framework. Psychological Bulletin, 141(3), 602-654. http: / / dx.doi.org/10.1037/a0038662

Cassibba, R., Coppola, G., Sette, G., Curci, A., \& Costantini, A. (2017). The transmission of attachment across three generations: A study in adulthood. Developmental Psychology, 53(2), 396-405. http://dx.doi.org/10.1037/ dev0000242

Chiorino, V., Roveraro, S., Caterina Cattaneo, M., Salerno, R., Macchi, E. A., Bertolucci, G. G., . . .Fernandez, I. (2016). A model of clinical intervention in the maternity ward: The breastfeeding and bonding EMDR protocol. Journal of EMDR Practice and Research, 10(4), 275-291. http://dx.doi.org/10.1891/1933-3196.10.4. 275

De Divitiis, A. M., \& Luber, M. (2016). EMDR therapy protocol for the prevention of birth trauma and postpartum depression in the pregnant woman. In $\mathrm{M}$. Luber (Ed.), Eye movement desensitization and reprocessing (EMDR) therapy scripted protocols and summary sheets: Treating anxiety, obsessive-compulsive, and mood-related conditions (pp. 325-350). New York, NY: Springer Publishing.
Doi, T. (1971). The anatomy of dependence. The key analysis of Japanese behavior. (J. Bester, Trans.). Tokyo/New York/ London: Kodansya International.

Dutra, L., Bureau, J. F., Holmes, B., Lyubchik, A., \& LyonsRuth, K. (2009). Quality of early care and childhood trauma: A prospective study of developmental pathways to dissociation. The Journal of Nervous and Mental Disease, 197(6), 383-390. http://dx.doi.org/10.1097/ NMD.0b013e3181a653b7

Edwards, A., Shipman, K., \& Brown, A. (2005). The socialization of emotional understanding: A comparison of neglectful and nonneglectful mothers and their children. Child Maltreatment, 10(3), 293-304. http:/ / dx.doi. org/10.1177/1077559505278452

George, C., Kaplan, N., \& Main, M. (1985). Adult attachment interview protocol. Unpublished manuscript. Berkeley: University of California.

Gomez, A. M. (2012). Healing the caregiving system: Working with parents within a comprehensive EMDR treatment. Journal of EMDR Practice and Research, 6(3), 136-144. http: / / dx.doi.org/10.1891/1933-3196.6.3.136

Gonzalez, A., Mosquera, D., Leeds, A. M., Knipe, J., \& Solomon, R. (2012). The AIP model and structural dissociation: A proposal to extend the framework. In A. Gonzalez \& D. Mosquera (Eds.), EMDR and dissociation: The progressive approach (pp. 31-50). Charleston, SC: Amazon Imprint.

Hanson, J. L., Nacewicz, B. M., Sutterer, M. J., Cayo, A. A., Schaefer, S. M., Rudolph, K. D., . . Davidson, R. J. (2015). Behavioral problem after early life stress: Contributions of the hippocampus and amygdala. Biological Psychiatry, 77(4), 314-323. http://dx.doi.org/10.1016/j.biopsych. 2014.04.020

Klaus, M. H., Kennell, J. H., \& Klaus, P. H. (1995). Bonding: Building the foundations of secure attachment and independence. New York, NY: Perseus Publishing.

Knipe, J. (2010). Dysfunctional positive affect: To assist clients with unwanted avoidance defenses. In $\mathrm{M}$. Luber (Ed.), Eye movement desensitization and reprocessing (EMDR) scripted protocols: Special populations (pp. 451-452). New York, NY: Springer Publishing Company.

Leeds, A. (2001). Guideline and procedures for EMDR resource development and installation. In F. Shapiro (Ed.), Eye movement desensitization and reprocessing, basic principles, protocols and procedures (pp. 434-440). New York, NY: The Guilford Press.

Leeds, A. (2013). Affect phobias in EMDR therapy: Developing affect tolerance capacities in client and clinician. The Handout of EMDRIA Annual Meeting 2013 in Austin, TX.

Leeds, A. (2015). Restructuring defenses, affects, self and other: Evolution in information processing model and method. Advanced Workshop Tokyo.

Leeds, A. (2016). A guide to the standard EMDR protocols for clinicians, supervisors, and consultants (2nd ed.). New York, NY: Springer Publishing Company. 
Lyons-Ruth, K., Pechtel, P., Yoon, S. A., Anderson, C. M., \& Teicher, M. H. (2016). Disorganized attachment in infancy predicts greater amygdala volume in adulthood. Behavioural Brain Research, 308, 83-93. http://dx.doi. org/10.1016/j.bbr.2016.03.050

Madrid, A. (2007). Repairing maternal-infant bonding failures. In F. Shapiro, F. W. Kaslow, \& L. Maxfield (Eds.), Handbook of EMDR and family therapy processes (pp. 131145). New York, NY: Wiley.

Madrid, A., Skolek, S., \& Shapiro, F. (2006). Repairing failures in bonding through EMDR. Clinical Case Studies, 5(4), 271-286. http:/ / dx.doi.org/10.1177/ 1534650104267403

Main, M., \& Solomon, J. (1986). Discovery of a new insecure-disorganized/ disoriented attachment pattern. In T. B. Brazelton \& M. Yogman (Eds.), Affective development in infancy (pp. 95-124). Norwood, NJ: Ablex.

Malik, N. M. (2012). The challenging child. Emotional dysregulation and aggression. In S. J. Summers \& R. Chanzan-Cohen (Eds.), Understanding early childhood mental health: Practical guide for professionals (pp. 25-39). Baltimore, MD: Paul H. Brookes Publishing.

Montag, C., \& Panksepp, J. (2017). Primary emotional systems and personality: An evolutionary perspective. Frontiers in Psychology, 8(i), 1-14. http://dx.doi.org/10. 3389/fpsyg.2017.00464

Nemeroff, C. B. (2004). Neurobiological consequences of childhood trauma. The Journal of Clinical Psychiatry, 65(Suppl 1), 18-28.

Okawara, M. (2010). Integration of family therapy and EMDR-How to apply to improve family relationship-. Japanese Journal of EMDR Research \& Practice, 2(1), 27-37.

Okawara, M. (2012). Psychotherapy for sibling abuse with 4th grade boy and his mother. Japanese Journal of EMDR Research \& Practice, 4(1), 41-50(Japanese).

Okawara, M. (2015). Emotional regulation and psychotherapy with children. Tokyo: Nihon Hyoron-Sya (Japanese).

O'Shea, K. (2009). The early EMDR trauma protocol. In R. Shapiro (Ed.), EMDR solution II: For depression, eating disorders, performance, and more (pp. 313-334). New York, NY: Norton Professional Books.

O’Shea, K., \& Paulsen, SL. (2007). "A protocol for increasing affect regulation and clearing early trauma". Paper presented at the: Eye Movement Desensitization o Reprocessing International Association Conference in Dallas, Texas.

Panksepp, J. (1998). Affective neuroscience; The foundation of human animal emotions. New York, NY: Oxford University Press.

Paulsen, S., \& Lanius, U. (2009). Toward an enbodied self. Integrating EMDR with somatic and ego state interventions. In R. Shapiro (Ed.), EMDR solution II: For depression, eating disorders, performance, and more (pp. 335-338). New York, NY: Norton Professional Books.

Paulsen, S. L. (2014). Temporal integration of early trauma and neglect. In U. L. Lanius, S. L. Paulsen, \& F. Corrigan (Eds.), The neurobiology and treatment of traumatic dissociation: Toward an embodied self (pp. 423-445). New York, NY: Springer Publishing.

Paulsen, S. L. (2017). When there are no words: Healing early trauma and neglect in the attachment period with EMDR therapy. Charleston, SC: Create Space.

Perry, B. D., \& Pollard, R. (1998). Homeostasis, stress, trauma, and adaptation. A neurodevelopmental view of childhood trauma. Child and Adolescent Psychiatric Clinics of North America, 7(1), 33-51.

Petzoldt, J., Wittchen, F. E., \& Martini, J. (2015). Maternal anxiety versus depressive disorders: Specific relations to infants' crying, feeding and sleeping problems. Child: Care, Health \& Development, 42(2), 231-245.

Popky, A. J. (2005). DeTUR, an urge reduction protocol for addictions and dysfunctional behaviors. In R. Shapiro (Ed.), EMDR solutions: Pathways to healing (pp. 167-188). New York, NY: W. W. Norton.

Porges, S. W. (2007). The polyvagal perspective. Biological Psychology, 74(2), 116-143. http:/ / dx.doi.org/10.1016/j. biopsycho.2006.06.009

Rosso, A. M., \& Airaldi, C. (2016). Intergenerational transmission of reflective functioning. Frontiers in Psychology, 7(1134), 1-11. http://dx.doi.org/10.3389/fpsyg.2016. 01903

Schore, A. N. (2003). Affect dysregulation \& disorder of the self. New York, NY: W. W. Norton.

Schuder, M. R., \& Lyons-Ruth, K. (2004). Hidden trauma' in infancy: Attachment, fearful arousal, and early dysfunction of the stress response system. In D. Osofsky Joy (Ed.), Young children and trauma: Intervention and treatment (pp. 69-104). New York, NY: Guilford Press.

Shapiro, F. (1995). Eye movement desensitization and reprocessing, basic principles, protocols and procedures. New York, NY: Guilford Press.

Shapiro, F. (2001). Eye movement desensitization and reprocessing, basic principles, protocols and procedures (2nd ed.). New York, NY: Guilford Press.

Stern, D. N. (1985). The interpersonal world of the infant: A view from psychoanalysis and developmental psychology. New York, NY: Basic Books.

Teicher, M. H., Andersen, S. L., Polcari, A., Anderson, C. M., Navalta, C. P., \& Kim, D. M. (2003). The neurobiological consequences of early stress and childhood maltreatment. Neuroscience \& Biobehavioral Reviews, 27(1-2), 3344. http: / / dx.doi.org/10.1016/S0149-7634(03)00007-1

Trevarthen, C. (1998). The concept and foundations of child intersubjectivity. In S. Bråten (Ed.), Intersubjective communication and emotion in early ontogeny (pp. 15-46). Cambridge, UK: Cambridge University Press.

Tucker, D. O. N. M., Luu, P., \& Derryberry, D. (2005). Love hurts: The evolution of empathic concern through the encephalization of nociceptive capacity. Development and Psychopathology, 17(03), 2005. http://dx.doi.org/10. 1017 /S0954579405050339

van der Hart, O., Nijenhuis, E. R. S., \& Solomon, R. (2010). Dissociation of personality in complex trauma-related disorder and EMDR: Theoretical consideration. Journal 
of EMDR Practice and Research, 4(2), 76-92. http://dx. doi.org/ 10.1891/1933-3196.4.2.76

van der Kolk, B. A. (2005). Developmental trauma disorder: Toward a rational diagnosis for children with complex trauma histories. Psychiatric Annals, 35(5), 401-408. http: / / dx.doi.org/ 10.3928/00485713-20050501-06

van IJzendoorn, M. H. (1995). Adult attachment representations, parental responsiveness, and infant attachment: A meta-analysis on the predictive validity of the adult attachment interview. Psychological Bulletin, 117(3), 387-403.

Verardo, A. R., \& Zaccagnino, M. (2016). Working on attachment issue with EMDR therapy: The attachment protocol. In M. Luber (Ed.), Eye movement desensitization and reprocessing (EMDR) therapy scripted protocols and summary sheets: Treating trauma and stressor-related conditions (pp. 45-81). New York, NY: Springer Publishing.

Wesselmann, D., Davidson, M., Armstrong, S., Schweitzer, C., Bruckner, D., \& Potter, A. E. (2012). EMDR as a treatment for improving attachment status in adults and children. Revue Européenne de Psychologie Appliquée/European Review of Applied Psychology, 62(4), 223-230. http: / / dx.doi.org/10.1016/j.erap.2012.08.008

Wesselmann, D., Schweitzer, C., \& Armstrong, S. (2016). Child attachment trauma protocol. In M. Luber (Ed.),
Eye movement desensitization and reprocessing (EMDR) therapy scripted protocols and summary sheets: Treating trauma-and stressor-related conditions (pp. 99-4343). New York, NY: Springer Publishing.

Zaccagnino, M., \& Cussino, M. (2013). EMDR and parenting: A clinical case. Journal of EMDR Practice and Research, 7(3), 154-166. http://dx.doi.org/10.1891/ 1933-3196.7.3.154

Zaccagnino, M., \& Verardi, A. R. (2016). Working on attachment issue with EMDR therapy: The attachment protocol. In M. Luber (Ed.), Eye movement desensitization and reprocessing (EMDR) therapy scripted protocols and summary sheets: Treating trauma-and stressor-related conditions (pp. 45-81). New York, NY: Springer Publishing.

Zeanah, C. H., \& Zeanah, P. D. (1989). Intergenerational transmission of maltreatment: Insights from attachment theory and research. Psychiatry, 52(2), 177-196. http: / / dx.doi.org/ 10.1080/00332747.1989.11024442

Correspondence regarding this article should be directed to Mii Okawara, PhD, Tokyo Gakugei University, 4-1-1, Nukui-kitamati, Koganei-shi, Tokyo, Japan 184-8501. E-mail: mii@u-gakugei.ac.jp 